\title{
Micropatterning of Functional Film by Liquid Phase Laser Direct Patterning Method
}

\author{
A. Watanabe ${ }^{1}$, E. Ohta ${ }^{2}$ and A. Shimofuku ${ }^{2}$ \\ ${ }^{1}$ Institute of Multidisciplinary Research for Advanced Materials, \\ Tohoku University, 2-1-1 Katahira, Aoba-ku, Sendai 980-8577, Japan \\ ${ }^{2}$ Technology Strategy Center, GJ Design \& Development Division, Ricoh Co., Ltd \\ 1005 Shimo-Ogino, Atsugi, Kanagawa 243-0298, Japan.
}

Keywords: liquid Phase laser direct patterning, micropatterning, lead zirconate titanate, micro-Raman spectroscopy

\section{Introduction}

The sol-gel process has been extensively studied because of the low cost and simple manufacturing process compared to the other processes such as CVD, sputtering, and laser ablation methods. The sol-gel process has been applied to prepare the film of a metal oxide with perovskite structure such as titanate ceramics such as lead zirconate titanate $\mathrm{Pb}_{\mathrm{x}} \mathrm{Zr}_{1-\mathrm{x}} \mathrm{TiO}_{3}$ (PZT), barium titanate $\mathrm{BaTiO}_{3}(\mathrm{BT})$ and so on. These inorganic films have potentials of the application to microelectronic devices widely. For example, the PZT has been intensively studied in recent years because of a variety of applications due to the high dielectric, ferroelectric, and piezoelectric properties. Although the sol-gel process is an attractive low cost and simple solution process, the sol-gel derived inorganic film is usually amorphous. Because the crystallization of the sol-gel derived PZT film requires a high heat treatment temperature above $700^{\circ} \mathrm{C}$, the substrate which has lower degradation temperature than the crystallization temperature cannot be applicable. In the previous papers, we have reported the low temperature crystallization by laser direct writing method using sol-gel derived precursor films [1-3]. This method has an additional advantage that crystalline micropaterns can be fabricated by scanning the laser beam on the sol-gel derived precursor film without photolithography and dry etching processes. One of the problems of the sol-gel process for the preparation of inorganic film is the crack formation, which is caused by shrink of the film eliminating organic moieties during the mineralization by heat treatment. The multistep process repeating the spin-coating and pre-heating is required to obtain a precursor film without cracking even for the precursor film of the laser processing. In this paper, we report the fabrication of a PZT micropattern by liquid phase laser direct patterning (LLDP) method. The LLDP method is a new approach to fabricate a crystalline micropattern on a substrate from liquid phase directly by scanning irradiation of laser beam focused on the substrate, which gives a possibility to prepare a crystalline micropattern on low heat-resistant substrates such as glass and plastic without cracking.

\section{Experimental}

A PZT precursor solution was prepared by following procedures. The starting materials were lead (II) acetate trihydrate $\mathrm{Pb}\left(\mathrm{OCOCH}_{3}\right)_{2}$. $3 \mathrm{H}_{2} \mathrm{O}$, zirconium tetra-n-propoxide $\mathrm{Zr}(\mathrm{O}-\mathrm{n}-\mathrm{Pr})_{4}$ and titanium tetra iso-propoxide $\mathrm{Ti}(\mathrm{O}-\mathrm{i}-\mathrm{Pr})_{4}$. Lead (II) acetate trihydrate was dissolved into 2-methoxyethanol and then zirconium tetran-propoxide and titanium tetra iso-propoxide were added with a total concentration of $0.5 \mathrm{M}$. The experimental setup of the liquid phase laser direct patterning of a PZT micropattern is illustrated in Fig.1. A Teflon vessel was filled with the precursor solution. A silicon substrate was immersed into the precursor solution in the Teflon vessel, and then sealed with a transparent glass substrate, a

Received April 1,2011

Accepted May 13, 2011 
separator, and Teflon screw ring. As laser sources, CW (continuous wave) DPSS (diode-pumped solid state) lasers $(532,405 \mathrm{~nm}$ ) were used. The laser beam was focused on the interface between the sol-gel solution and the substrate through optical microscope (OLYMPUS BX 51) equipped with an objective lens. The focused laser beam was scanned by a computer-controlled xyz stage.

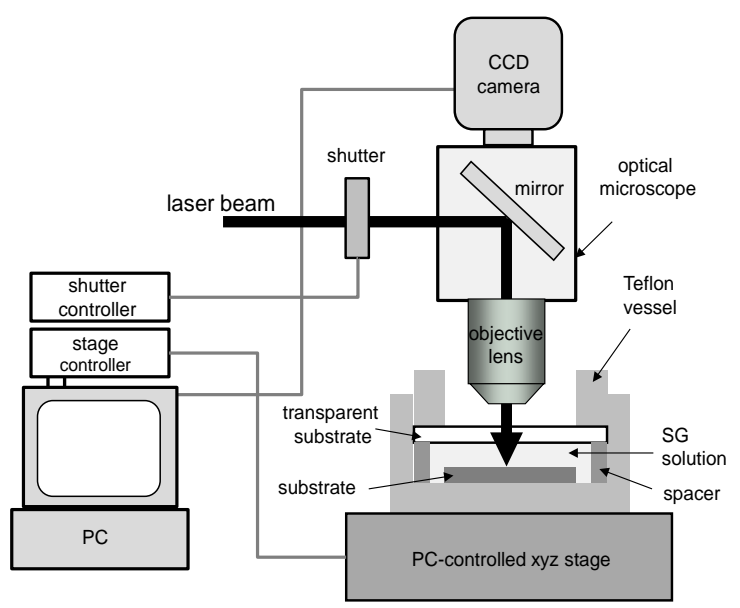

Fig.1. Experimental setup for LLDP method.

\section{Results and discussion}

The laser focusing on the Si substrate immersed into the precursor solution caused the bubble formation as shown in Fig.2. The moving of the bubble with scanning laser beam was observed.

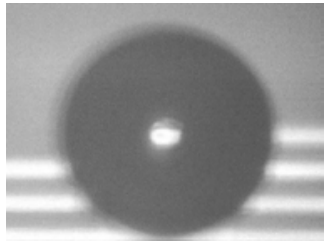

Fig.2. Bubble formed at laser focusing point.

Fig.3a shows the 3D image of PZT micro-lines by a color 3D laser scanning microscope (K eyence VK -9700), where 532-nm laser beam was focused on a Si substrate through a glass window and the precursor solution with the scanning interval of 10 $\mu \mathrm{m}$. Because the precursor solution has no absorption at the wavelength of laser beam, the heating of the Si substrate is caused by laser irradiation, which induces the deposition and the crystallization of PZT film.

The fabrication of a PZT film on a transparent glass substrate was possible by focusing $405-\mathrm{nm}$ laser beam on the interface between the glass substrate and the precursor solution through the glass substrate, where the laser beam is absorbed by the absorption edge of the precursor solution. In this case, the formation of a PZT film thicker than $10 \mu \mathrm{m}$ was possible as shown in Fig. $3 \mathrm{~b}$.

(a)

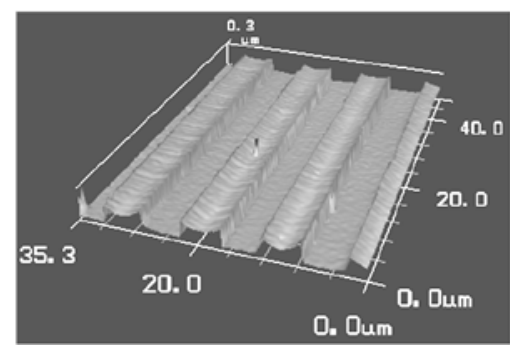

(b)

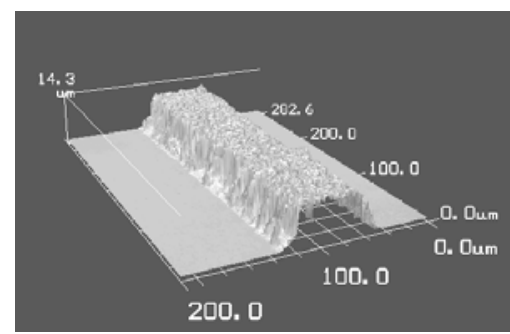

Fig.3. 3D image of PZT patterns by LLDP method on Si (a) and glass (b) substrates.

The crystal structure of PZT pattern was investigated by micro-Raman spectroscopy. Fig.4 shows the Raman spectrum of PZT pattern as shown in Fig.3b. The broad Raman band at around $550 \mathrm{~cm}^{-1}$ suggests the perovskite structure.

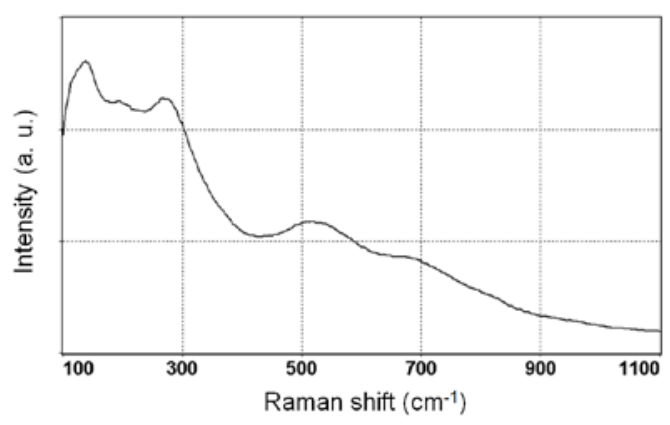

Fig.4. M icro-Raman spectrum of PZT pattern.

\section{References}

1. A. Watanabe, T. Tanase, Y. Kobayashi, M. K onno, S. Yamada, and T. M iwa, J pn. J. Appl. Phys., 42, L843 (2003).

2. Y. K obayashi, K. M iyajima, D. Nagao, A. Watanabe, S. Yamada, T. Miwa, and M. Konno, Applied Surface Science, 253, 5293 (2007).

3. A. Watanabe, T. Tanase, K. M iyajima, D. Nagao, Y. K obayashi, and M. K onno, Proc. SPIE, 7585, 75850N-1 (2009). 